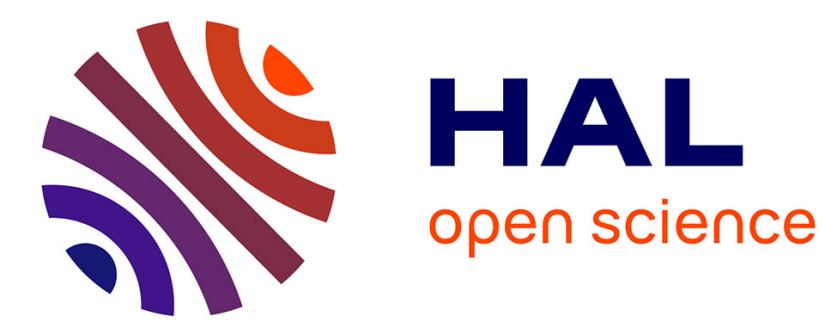

\title{
Sacrifices d'animaux à l'Hérakleion de Thasos
}

\author{
Anne Pariente, Jacques Des Courtils, Armelle Gardeisen
}

\section{To cite this version:}

Anne Pariente, Jacques Des Courtils, Armelle Gardeisen. Sacrifices d'animaux à l'Hérakleion de Thasos. Bulletin de Correspondance Hellenique, 1996, 120 (2), pp.807-827. 10.3406/bch.1996.4628 . hal-01415520

\section{HAL Id: hal-01415520 \\ https://hal.science/hal-01415520}

Submitted on 13 Dec 2016

HAL is a multi-disciplinary open access archive for the deposit and dissemination of scientific research documents, whether they are published or not. The documents may come from teaching and research institutions in France or abroad, or from public or private research centers.
L'archive ouverte pluridisciplinaire HAL, est destinée au dépôt et à la diffusion de documents scientifiques de niveau recherche, publiés ou non, émanant des établissements d'enseignement et de recherche français ou étrangers, des laboratoires publics ou privés. 
Anne Pariente Jacques Des Courtils Armelle Gardeisen

\section{Sacrifices d'animaux à l'Hérakleion de Thasos}

In: Bulletin de correspondance hellénique. Volume 120, livraison 2, 1996. pp. 799-820.

Citer ce document / Cite this document :

Pariente Anne, Des Courtils Jacques, Gardeisen Armelle. Sacrifices d'animaux à l'Hérakleion de Thasos. In: Bulletin de correspondance hellénique. Volume 120, livraison 2, 1996. pp. 799-820.

doi : $10.3406 /$ bch.1996.4628

http://www.persee.fr/web/revues/home/prescript/article/bch_0007-4217_1996_num_120_2_4628 


\section{Abstract}

An archaeozoological analysis was carried out in the Herakleion on Thasos in order to compare the textual evidence concerning the «ritual of the Prytany» with that yielded by recent excavations, which in a dear stratified context produced securely dated pottery in association with abundant well-preserved osteological material. The results show that if the ritual, or at least the dietary prohibitions and methods of dividing up the carcasses connected with the ritual were ob served in the Archaic period, this observance was not kept up in later rimes. Furthermore the duality of the cuit of Herakles, heroic and divine, was confirmed on Thasos by the convergence of the archaeological and textual evidence and the faunal remains.

\section{Résumé}

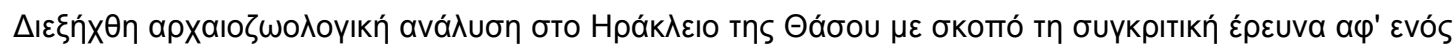

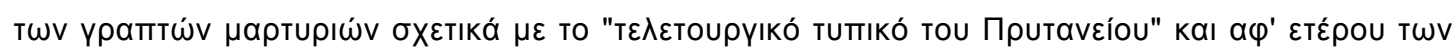

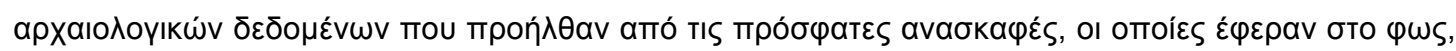

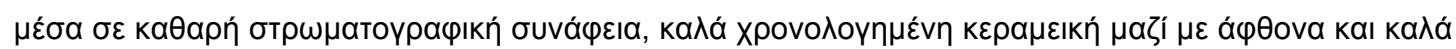

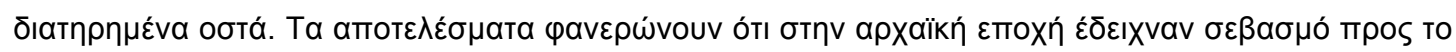

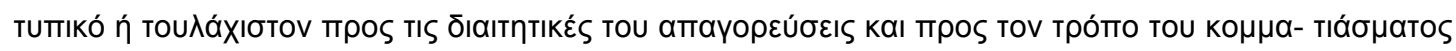

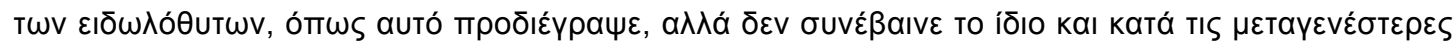

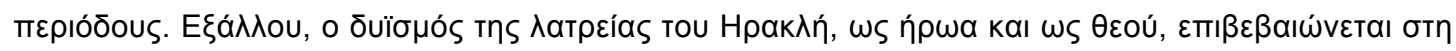

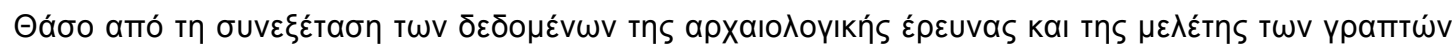

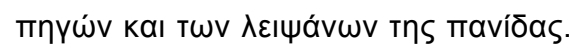

$\pi \varepsilon \rho i ́ \lambda \eta \psi \eta$

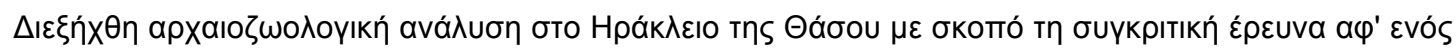

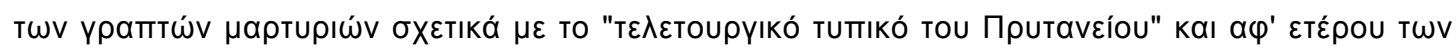

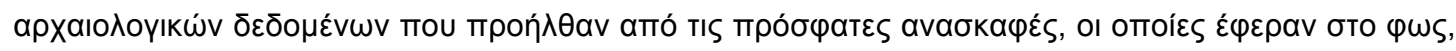

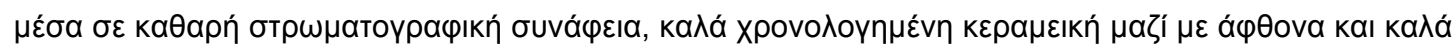

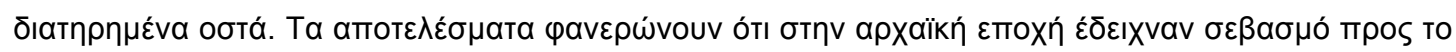

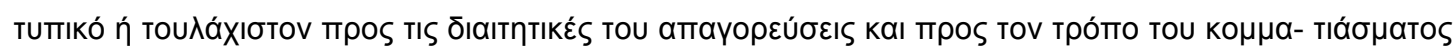

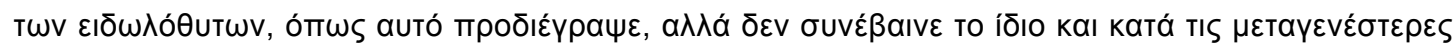

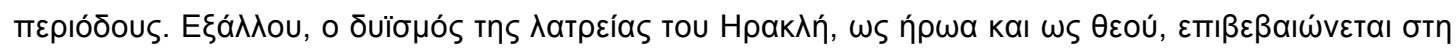

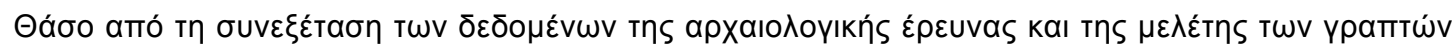

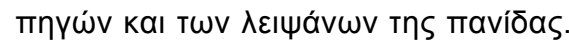




\section{Sacrifices d'animaux à l'Hérakleion de Thasos*}

\section{Présentation}

Si les découvertes archéologiques accumulées au cours des temps ont porté à notre connaissance un nombre non négligeable de règlements cultuels inscrits sur pierre, elles ont rarement fourni la possibilité de vérifier leur observance par les anciens. Le site de Thasos présente cette particularité, puisque la découverte déjà ancienne sur l'agora d'une inscription concernant le culte d'Héraklès (le "rituel du prytanée ")' a été complétée par la fouille et la publication du sanctuaire d'Héraklès, effectuées par M. Launey au milieu de ce siècle ${ }^{2}$. Plus récemment, la reprise des fouilles de ce sanctuaire a permis la collecte de nombreux ossements animaux ${ }^{3}$, dont on lira ci-après l'étude.

Lintérêt de ces ossements, trouvés dans un contexte stratigraphique clair, en association avec des céramiques bien datées et des constructions identifiables (temple, autel...), est d'abord dû à la possibilité qui est ainsi procurée de les confronter avec un texte rituel. Mais leur intérêt consiste aussi à apporter un argument dans le débat ancien sur la nature " ontologique " d'Héraklès tel qu'il était adoré à Thasos.

Les éléments archéologiques, épigraphiques et religieux du débat ont déjà été exposés et discutés à plusieurs reprises, notamment par deux d'entre nous en $1991^{4}$ : à cette occasion, nous avions fait une première présentation des ossements découverts à l'Hérakleion thasien. L'étude qu'on va lire est plus systématique et permet d'aboutir à quelques constatations importantes, dont nous voudrions souligner la portée.

Comme on le sait, le règlement sacrificiel d'Héraklès thasien interdit explicitement le sacrifice de la chèvre et du porc, mais aussi la partition et la manducation des viandes: il ne laisse donc place qu'à des sacrifices holocaustiques.

L'étude d'A. Gardeisen montre qu'à l'époque archaïque, on ne trouve ni chèvre ni porc parmi les animaux sacrifiés à l'Hérakleion : les interdits rituels sont donc observés. En revanche il s'en trouve dans les restes sacrificiels du IV $v^{e}$. (lot $\left.n^{\circ} 6\right)^{5}$. Doit-on supposer un abandon du rituel ? Le fait est curieux. 
Le problème se complique si 'on ajoute qu'un bon nombre d'ossements découverts à l'Hérakleion portent des traces de partition des animaux et de découpage, supposant une consommation des viandes : ce fait est en contradiction absolue avec le texte du rituel sacrificiel du prytanée, qui interdit le découpage des animaux et requiert l'holocauste... Tout montre au contraire que l'on banquetait en l'honneur d'Héraklès, ce qui confirme l'identification dc l'édifice "aux oikoi" (appellation due à M. Launey) à un hestiatorion banal.

Ainsi les ossements retrouvés à l'Hérakleion de Thasos montrent que la pratique cultuelle s'y apparentait aux cultes antiques les plus normaux voués à une entité divine : on sacrifiait des animaux divers, on les partageait ensuite, certains morceaux étaient brûlés, d'autres mangés au cours de repas qui se déroulaient dans l'hestiatorion construit à cet effet dans l'enceinte du sanctuaire.

De plus, si l'on admet cette conclusion, en faveur de laquelle l'étude qu'on va lire offre une argumentation solide, on devra admettre l'existence à Thasos de deux rituels différents en l'honneur d'Héraklès: un rituel de type holocaustique-héröque, attesté par le texte "du prytanée " et un rituel divin normal attesté par les ossements animaux retrouvés à l'Hérakleion et par la présence d'un hestiatorion dans le sanctuaire. On peut assurément mettre en doute la pertinence générale de la distinction entre cultes héroïques et cultes divins, qui n'est pas toujours nette: il n'en est pas moins vrai que les témoignages archéologiques recueillis à Thasos révèlent l'existence des deux rituels différents. Le fameux texte d'Hérodote (II 44) soulignant que le héros Héraklès était paré à Thasos d'une nature divine se trouve ainsi confirmé.

La localisation à l'Hérakleion de ce culte divin ne présente aucun problème, s'agissant d'un sanctuaire "normal", doté d'un temple, d'un autel et d'une salle de banquet. Plus délicate est la localisation du culte héroïque. Car Hérodote pense peut-être aux Thasiens lorsqu'il ajoute (ibid.): "J'estime très sage la conduite de ceux des Grecs qui ont dédié chez eux des sanctuaires à deux Héraklès, offrant à l'un, qu'ils appellent olympien, des sacrifices comme à un immortel, tandis quà l'autre ils offrent des sacrifices infernaux comme à un héros. " Or un deuxième sanctuaire, dédié au héros Héraklès, existait peutêtre bel et bien sur l'agora de Thasos: on en a pour indice un pied de table à offrande en marbre portant ses attributs (massue et léontè) et trouvé dans le même secteur de l'agora antique que la pierre portant le rituel hérö̈que "du prytanée " dont il a été question.

Cette hypothèse, pour séduisante qu'elle soit, ne peut actuellement devenir une certitude, puisquil n'y a ni conformité complète ni contradiction totale entre le rituel de l'agora et les constatations archéozoologiques faites dans le sanctuaire d'Héraklès... Elle laisse toutefois la porte ouverte à la possibilité, propre à Thasos, de deux cultes distincts d'Héraklès? 


\section{II. Étude archéozoologique}

Le matériel faunique exhumé lors des fouilles de l'Hérakleion de Thasos a été examiné en plusieurs lots déterminés en accord avec les archéologues, en fonction de leur origine archéologique et de leur nature.

Lot 1 : le premier lot (fouille 1985) est un dépôt d'ossements animaux associé à un ensemble céramique localisé au niveau de l'autel du premier temple (temple A daté du $3^{e}$ quart du VI $s$. av. J.-C.). Cette zone dite " aire sacrificielle » ou " autel " est comprise essenFG. 1 tiellement entre le mur Est du temple A et les fondations extérieures du temple B et correspond aux sondages P5, P6 et P7. D'après les observations archéologiques, il semble permis de considérer que ces accumulations d'ossements et de céramiques mêlées à un sédiment cendreux ont été déposées en une seule fois et forment un ensemble cohérent et complet.

Les couches archéologiques concernées sont les suivantes:

$\mathrm{N}^{\circ} 507$ et 508 (sondage P5)

$\mathrm{N}^{\circ} 527$ à 532 (sondage $\mathrm{P} 6$ )

$\mathrm{N}^{\circ} 661$ et 662 (sondage P7bis)

$N^{\circ} 668$ à 671 et $N^{\circ} 673$ (berme des sondages P6-P7)

$N^{\circ} 778$ et 779 (berme des sondages P5-P6).

Lot 2 : ce lot, lié à l'aire sacrificielle, a été isolé du précédent en raison de l'absence de céramique associée. Toutefois, il n’a pu être établi aucune association certaine, tant archéologique que chronologique, avec le premier lor. D'un point de vue faunique, les cinq couches archéologiques considérées $\left(\mathrm{N}^{\circ} 556,583,655,674,667\right)$ n'ont livré qu’un matériel pauvre et très fragmentaire difficile à caractériser malgré une dominance relative des ovinés-caprinés et la présence de traces de boucherie.

Lot 3 : le troisième lot est constitué par l'ensemble des couches issues des remblais de construction du second temple (temple B), daté du début du Ve siècle av. J.-C.

Il est représenté par les couches archéologiques suivantes:

$\mathrm{N}^{\circ} 533$ (sondage $\mathrm{P} 6$ )

$N^{\circ} 557$ (sondage naos 3)

$N^{\circ} 651$ à 653 et 656 (sondage P7)

$\mathrm{N}^{\circ} 660$ (sondage P7bis)

$\mathrm{N}^{\circ} 666$ (berme des sondages P6-P7)

$\mathrm{N}^{\circ} 753$ et 754 (sondage P8)

$\mathrm{N}^{\circ} 777$ et 780 (berme des sondages P5-P6).

Lot 4 : il s'agit d'un lot relativement hétérogène constitué par les niveaux de remplissage des sondages dits cours 2, 2bis, 3 et 3 bis, attenants au temple. Le contenu archéologique ne permet malheureusement pas de dater ces ensembles avec précision. Toutefois, sil n'est pas possible d'établir une contemporanéité certaine avec les couches de remblais 
Fig. 1. Plan de la fouille de l'Hérakleion de Thasos:

en gris foncé, le premier Hérakleion ; en gris clair, emplacement des sondages. Plan T. Koželj (EFA). 
du temple B (lot 3), cet ensemble reste datable de l'époque archaïque.

Les couches archéologiques analysées sont les suivantes:

$\mathrm{N}^{\circ} 601,602,609,610,611$ (cour 2)

$\mathrm{N}^{\circ} 605,606,608,612$ et 614 à 619 (cour 2bis)

$N^{\circ} 626$ à 630 et 639 (cour 3 )

$N^{\circ} 632$ à 636 et 638,640 (berme entre les cours 3 et 2 bis)

$\mathrm{N}^{\circ} 701$ à 705 (cour 3bis)

$N^{\circ} 706$ à 708 (berme entre les cours 3 et 3 bis).

Lot $5:$ le lot 5 présente un assemblage similaire à celui du lot $4:$ il correspond en effet au fonctionnement de la cour 4, mais en a été isolé du fait de son éloignement topographique. Il est représenté par les couches $\mathrm{N}^{\circ} 727,728,730$ et 731 .

Lot 6: le dernier lot est un ensemble plus récent, recueilli dans un sondage ouvert dans la cour au Sud de l'entrée du temple, où l'on a dégagé un dépôt de type "favissa" (?) : il est tout à fait différencié des précédents et les associations de céramiques le placent dans le courant du IV siècle av. J.-C. D'un point de vue faunique, ce lot s'apparente à une accumulation de type dépotoir, caractérisée par une bonne conservation des ossements issus de la couche unique $\mathrm{N}^{\circ} 2057$.

Devant cet éventail hétéroclite de contextes archéologiques, notre attention se portera principalement sur l'assemblage du lot 1 bien caractérisé, associé à la fois à une structure monumentale et à des données textuelles fournies par l'épigraphie (cf. introduction).

\section{Dépôt osseux de l'aire sacrificielle (lot 1)}

Le matériel faunique découvert en association avec les céramiques liées au culte hérakléen est relativement abondant et bien conservé. Sur un total de 235 restes osseux et dentaires, 225 ont été déterminés du double point de vue anatomique et spécifique. Cet ensemble témoigne de la présence d'au moins dix animaux dont huit sont des ovicaprinés (moutons ou chèvres) et deux sont des bœufs. Le spectre faunique se limite apparemment à ces deux espèces concernées par le rituel des sacrifices animaux?. En ce qui concerne les ovicaprinés, la différenciation spécifique entre moutons et chèvres n'a pu être établie systématiquement compte tenu des similitudes anatomiques et morphologiques de ces deux taxons au niveau ostéologique. Toutefois, si le mouton (Ovis aries L.) a pu être attesté sur un ensemble de 16 restes osseux, essentiellement des extrémités articulaires d'os longs (au nombre de 17 au total), aucun ossement n'a été attribué à la chèvre (Capra bircus L.). Bien que ce taux de diagnose spécifique soit faible, nous parlerons ici de moutons. Ce résultat s'explique en partie par le taux de fragmentation important du matériel qui nous a d'ailleurs engagée, par prudence, à considérer que certains éléments ne présentant pas de caractères morphologiques propres à l'une ou l'autre espèce étaient assimilés à des restes d'ovicaprinés notés: $\mathrm{O} / \mathrm{C}$ (tableau $1: \mathrm{NR}$ cf. $\mathrm{O} / \mathrm{C}=27$ ). 


\begin{tabular}{|c|c|c|c|c|c|c|c|c|c|c|c|c|c|c|c|c|c|c|}
\hline \multirow{2}{*}{$\begin{array}{l}\text { Hérakleion } \\
\text { Faune }\end{array}$} & \multicolumn{3}{|c|}{ LOT 1} & \multicolumn{3}{|c|}{ LOT 2} & \multicolumn{3}{|c|}{ LOT 3} & \multicolumn{3}{|c|}{ LOT 4} & \multicolumn{3}{|c|}{ LOT 5} & \multicolumn{3}{|c|}{ LOT 6} \\
\hline & NR & $\%$ NR & NMI & NR & $\%$ NR & NMI & NR & $\%$ NR & NMI & NR & $\%$ NR & NMI & NR & $\%$ NR & NMI & NR & $\%$ NR & NMI \\
\hline Mouton & 16 & & 8 & 2 & & 1 & 0 & & & 2 & & 1 & 0 & & & 13 & & 5 \\
\hline Chèvre & 0 & & 0 & 0 & & 0 & 0 & & & 0 & & 0 & 0 & & & 6 & & 4 \\
\hline O/C global & 145 & & 8 & 11 & & 2 & 20 & & 3 & 44 & & 5 & 27 & & 3 & 62 & & 9 \\
\hline cf. $0 / \mathrm{C}$ & 27 & & & 6 & & & 12 & & & 38 & & & 6 & & & 0 & & \\
\hline$O / C$ total & 172 & 76,4 & 8 & 17 & 77,3 & 2 & 32 & 64 & 3 & 82 & 72,6 & 5 & 33 & 78,6 & 3 & 62 & 60,2 & 9 \\
\hline Bœuf & 42 & & & 5 & & 1 & 14 & & & 20 & & 2 & 7 & & 1 & 18 & & 2 \\
\hline cf. Bos & 11 & & & 0 & & & 4 & & & 7 & & & 0 & & & 2 & & \\
\hline Bœuf total & 53 & 23,6 & 2 & 5 & 22,7 & 1 & 18 & 36 & 1 & 27 & 23,9 & 2 & 7 & 16,7 & 1 & 20 & 29,1 & 2 \\
\hline Équidés & 0 & & & 0 & & & 0 & & & 0 & & & 0 & & & 3 & 2,9 & 1 \\
\hline Porc & 0 & & & 0 & & & 0 & & & 2 & 1,8 & 1 & 1 & 2,4 & 1 & 18 & 17,5 & 4 \\
\hline Divers & 0 & & & 0 & & & 0 & & & 2 & 1,8 & $* * *$ & 1 & 2,4 & $* * *$ & 0 & & \\
\hline Total NRD & 225 & & & 22 & & 3 & 50 & & & 113 & & & 42 & & & 103 & & \\
\hline indét. & 10 & 4,3 & & 2 & 8,3 & & 5 & 10 & & 54 & 33,3 & & 1 & 2,4 & & 13 & 11,2 & \\
\hline TOTAL NR & 235 & & & 24 & & & 55 & & & 167 & & & 43 & & & 116 & & \\
\hline TOTAL NMI & & & 10 & & & 3 & & & 4 & & & 8 & & & 5 & & & 16 \\
\hline
\end{tabular}

Tableau 1. - Dénombrement de la faune terrestre de l'Hérakleion de Thasos.

NB : Divers désigne les témoins épars de la présence d'oiseau et de tortue. Pour plus de détail, cf. texte.

\section{a. Les moutons du dépôt sacrificiel}

Le nombre minimum d'individus estimé à partir des 172 restes de moutons est un NMI de combinaison établi selon le principe des appariements ou connexions potentiels en fonction de la taille et de l'état de croissance (âge) des ossements et des dents. Ainsi, huit individus ont été mis en évidence et montrent une population relativement variée : les classes d'abattage n'indiquent en effet aucun choix déterminé de l'animal en fonction de son âge.

Les classes d'âge ont été principalement établies sur le degré d'usure dentaire ${ }^{10}$ et plus rarement sur l'état de synostose des os longs ${ }^{\prime \prime}$. Les résultats obtenus indiquent la présence de :

-2 agneaux évalués à environ 3 mois ;

- 3 jeunes adultes dont un mâle et une femelle compris entre 18 et 24 mois ;

- 3 adultes dont deux de 4 ou 5 ans et le troisième d'environ 6-7 ans.

En terme de conservation anatomique, les représentations des différentes portions squelettiques, appréhendées globalement pour l'ensemble de la population ovine, montrent la présence de la quasi-intégralité de l'animal à l'exclusion des os du carpe, de l'ulna et de la patella. Leur déficit dans les courbes de représentation squelettiques peut s'expliquer par le mode de traitement des carcasses, en particulier pour l'articulation carpienne qui a pu être éliminée lors de la découpe au moment du dépouillage. Cependant, l'absence de la patella (ou rotule) qui est prise dans l'articulation du genou, entre fémur et tibia (ces derniers sont présents), est plus étonnante; on notera, en parallèle, une faible proportion 


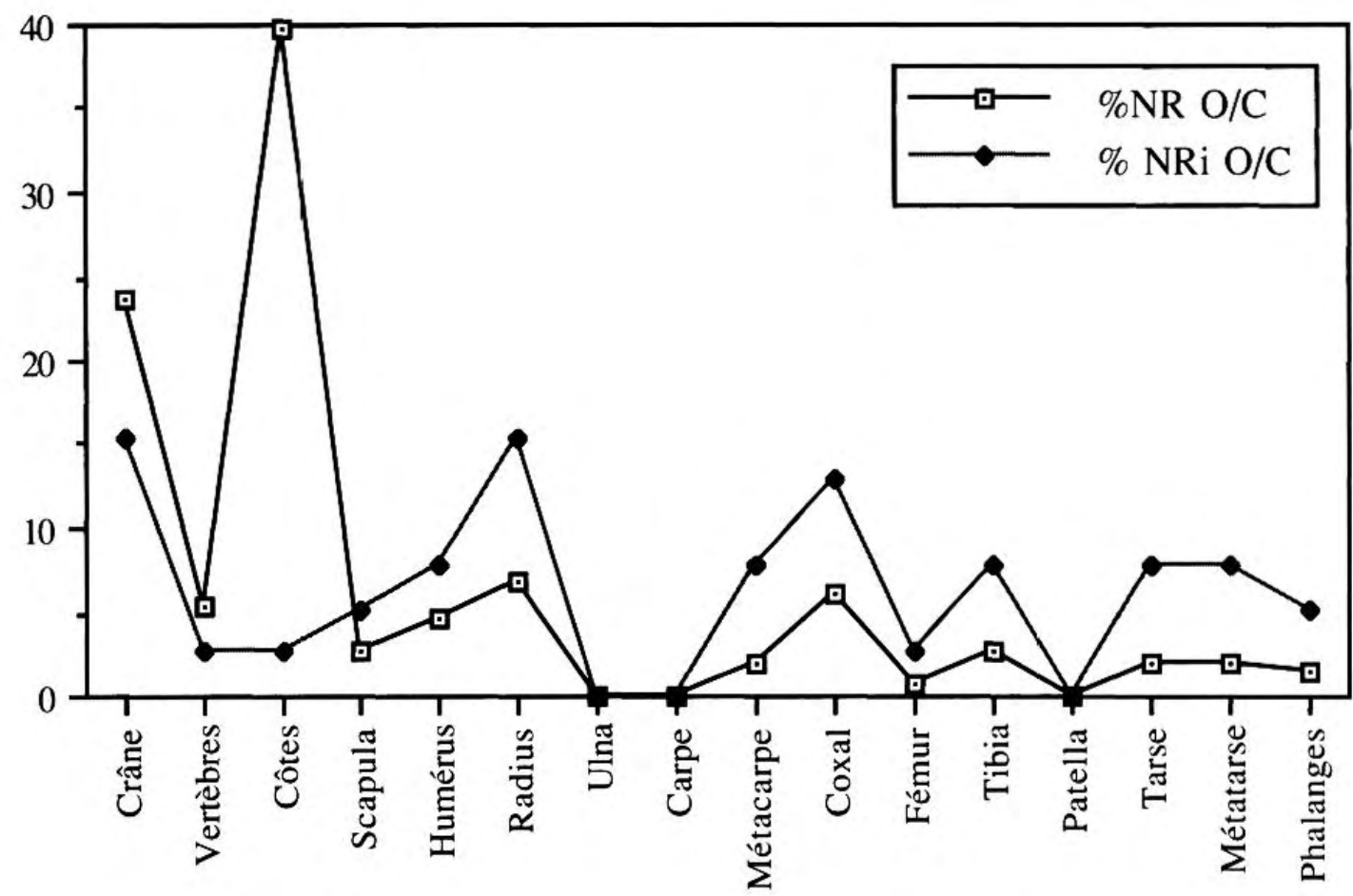

Fig. 2. Représentation anatomique des moutons de l'autel.

de fémurs qui laisserait supposer que le débitage de la cuisse pouvait également englober la partie supérieure du tibia (elle-même très mal représentée) : cette portion, une des plus charnues du squelette, a sans doute fait l'objet d'une exploitation différenciée et a pu être rejetée dans un autre lieu. Enfin, un autre argument pouvant justifier l'absence de ces os est leur petite taille, qui peut rendre difficile leur ramassage lors de la fouille.

Les courbes de représentation anatomique donnent une image directe de la conservation en nombre de restes (NR) d'une part et en nombre de reste initial (NRi) d'autre part. La vision en NR est uniquement tirée du décompte, pièce par pièce, de chacun des restes en fonction de leur nature (crâne, vertèbre, côte, scapula, etc.). On s'aperçoit vite que cette courbe, trop dépendante des données brutes et des phénomènes de fragmentation qui multiplient le nombre de restes, est faussée : en effet, les os fragiles comme les côtes et les crânes sont sur-représentés. C'est pour cette raison que nous avons eu recours à une estimation de nombre de reste initial (NRi) correspondant à une évaluation du nombre d'os entiers à partir de l'ensemble des fragments dénombrés. Cette approche permet de nuancer les observations précédentes et met en évidence le degré de fragmentation atteint par la différence plus ou moins prononcée entre courbe NR et courbe NRi. Il est dès lors possible de faire apparaître une dominance des restes du crâne, du radius, du coxal et dans une moindre mesure du tibia, du tarse et du métatarse; les extrémités des pattes sont peu nombreuses (cf. la représentation des phalanges). Bien qu'indicative, cette méthode n'en reste pas moins trop dépendante des données brutes soumises aux aléas de la conservation différentielle ostéologique. Le nombre d'ossements doit être mis en relation 
avec la population initiale : pour cela, nous avons eu recours à un calcul du taux de conservation (TC) établi à partir du nombre de moutons répertoriés. Le principe en est simple: si la population initiale (NMI) est de huit moutons, on doit théoriquement obtenir, pour chaque segment, un NMI de 8 dans l'hypothèse où les carcasses ont été exploitées et rejetées intégralement in situ. De la même manière, on calculera un NRi théorique attendu pour chaque os : sur une base de huit ovins, on attendra un NRi de 8 pour le crâne, de 16 pour les os symétriques (scapula, humérus, radius, ulna, coxal, fémur, tibia, métapodes...) ou de $8 \mathrm{X}$ n pour les ossements plus nombreux (n étant le nombre d'os présents dans un squelette complet).

Prenons pour exemple le NRi théorique des phalanges pour huit individus : $\mathrm{n}$ sera égal à 24, donc le NRi attendu sera de $24 \mathrm{X} 8$ soit 184 phalanges. Pour deux phalanges dénombrées dans l'assemblage osseux archéologique, on obtiendra un Taux de Conservation: TC NRi de $2 / 184 \times 100$. Si ces deux phalanges appartiennent au même individu, le TC NMI sera alors de $1 / 184$ X 100. Il arrive parfois que le NRi soit égal au NMI et les histogrammes se recoupent : c'est ici le cas des crânes, vertèbres, côtes et scapulas. En général, le taux de conservation sur le NMI surévalue la représentation de l'os du fait qu'un os isolé peut représenter un individu à lui seul. Cela explique la différence entre TC NRi et TC NMI pour le tarse ou les phalanges qui sont des os relativement nombreux dans le squelette.

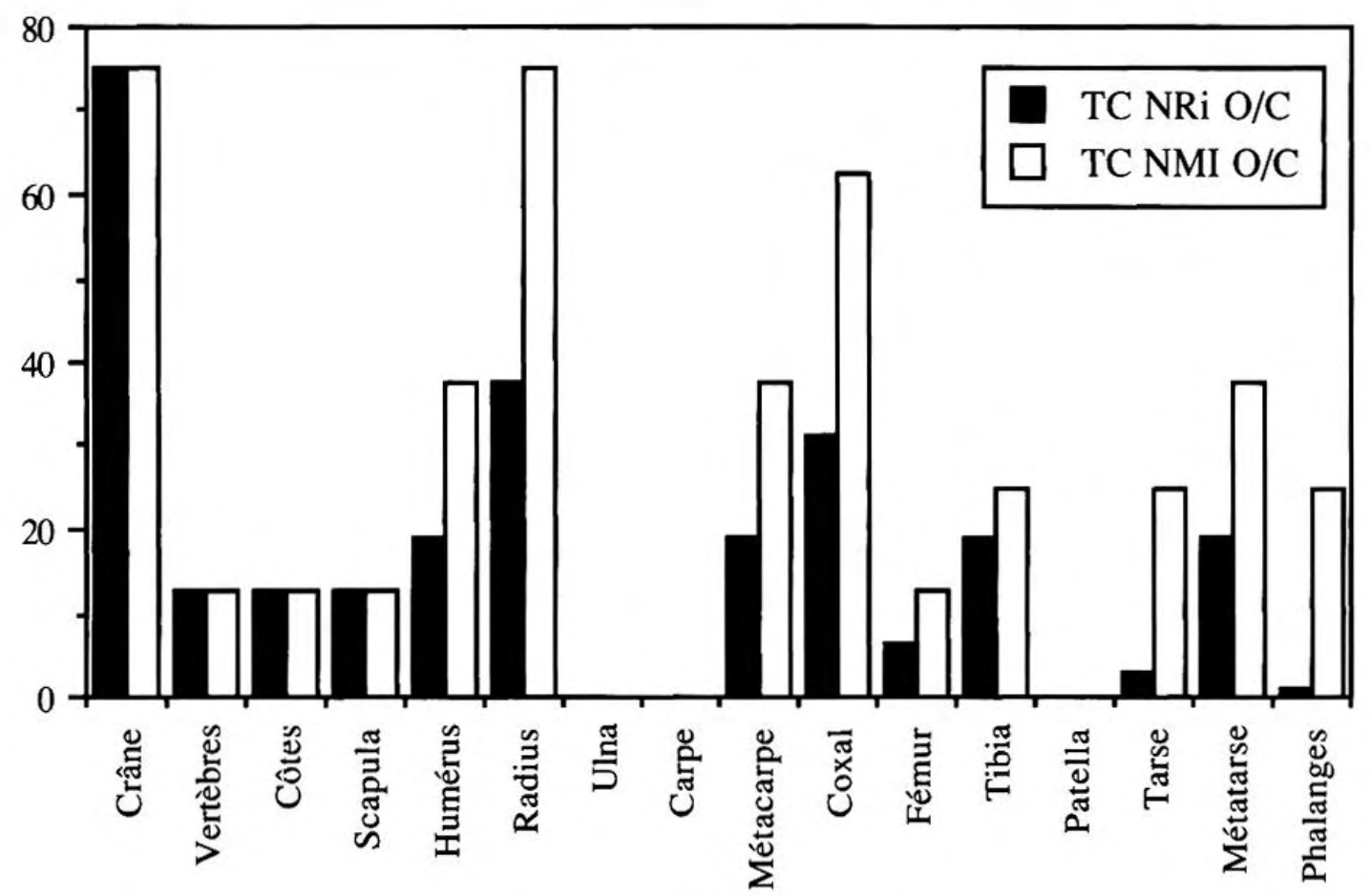

Fig. 3. Taux de conservation des moutons de l'autel. 
Nous considérons que le taux de conservation calculé sur la base du NRi est le plus proche de la réalité dans la mesure où il peut permettre de rendre compte du partage de l'animal : un membre (conçu comme une suite d'ossements) ou une partie de membre (un os isolé) peut être consommé ou sacrifié, et pas l'autre...

Ainsi, les taux de conservation mettent en évidence des variations sensibles d'un os à l'autre qui répondent aux actions de divers facteurs:

- l'intervention anthropique sur les carcasses que l'on peut appréhender par les représentations relatives des différents ossements et l'analyse des traces de découpe ;

- les phénomènes de conservation différentielle qui privilégient la conservation de certains os ou portions osseuses du fait de leur robustesse naturelle et de leur constitution : les parties compactes, épaisses et denses résistent davantage aux perturbations et phénomènes de tassement des terres que les portions fines ou spongieuses;

- les éventuelles modifications post-dépositionnelles que peuvent entraîner le piétinement, le remaniement des terres par l'action d'agents extérieurs tels que des animaux (fouisseurs, carnivores), ou encore par des phénomènes d'altération physico-chimique.

Toutes ces manifestations d'ordre archéozoologique ou taphonomique sont à l'origine de la modification de l'assemblage initial qui, par hypothèse, est un squelette complet.

Le profil de conservation des moutons de l'aire sacrificielle témoigne d'une certaine hétérogénéité dans le sens où il n'apparaît pas de choix spécifiques de portions anatomiques. Outre l'absence de certains ossements déjà évoquée, les éléments prédominants sont les crânes (essentiellement représentés par des hémi-mandibules), les radius et coxaux, puis dans une moindre mesure les extrémités distales des humérus ou de tibias et enfin les extrémités proximales des métapodes. Globalement, on peut considérer que ces ossements correspondent à des parties peu charnues. À l'inverse, le rachis (côtes et vertèbres), la scapula (palette) avec éventuellement l'humérus (épaule), le fémur (gigot) présentent des taux relativement faibles: trois épaules de mouton et un seul gigot alors que, théoriquement, on en attendait seize de chaque. On peut alors en déduire que les parties manquantes ont fait l'objet d'un traitement différencié.

De la même manière, les bases des pattes, à savoir les doigts représentés anatomiquement par la base des métapodes et les phalanges, sont quasiment absentes. L'observation de stries circulaires de découpe à la base d'un métacarpe suggère une opération de dépouillage qui aurait entraîné l'élimination des phalanges avec la peau. Cette répartition évoque une mise en quartier à laquelle succède une certaine dispersion des restes, qui pourrait trouver une explication dans une éventuelle distribution ou un partage des carcasses en morceaux destinés à des traitements différents.

A ce niveau, l'analyse des marques d'exploitation anthropique (impacts de découpe, stries de découpe, de désarticulation ou de décharnement) va permettre d'affiner l'interprétation de l'assemblage. Dans l'ensemble, 21,7\% des restes osseux présentent des 
TABLEAU 2

FIG. 4

FIG. 5

traces d'activités bouchères dont nous donnons le détail en fonction de leur localisation sur diaphyse ou articulation dans un tableau récapitulatif. La fréquence des traces observées par portion osseuse est exprimée dans la figure "Nombre de restes porteurs de marques de découpe par ossement de moutons "; leur localisation et leur nature sont indiquées sur un schéma.

Crâne: l'essentiel des restes crâniens est représenté par des mandibules ou maxillaires porteurs de séries jugales alors que le neurocrâne, plus fragile, n'a pas résisté au temps. L'enlèvement de la corne, de même que la découpe d'une cheville osseuse, est visible, ce qui suppose une utilisation artisanale probable de ces deux éléments. Par ailleurs, on note une strie de découpe circulaire du museau au niveau d'un incisif supérieur (ou pré-maxillaire) : cette opération est certainement en relation avec le dépouillage des animaux, déjà évoqué par l'absence des phalanges. Enfin, deux stries profondes et longitudinales localisées sur un fragment de temporal et occipital pourraient évoquer une découpe du crâne en deux destinée à la récupération de la cervelle (?). L'attrait alimentaire de la tête est également démontré par les marques de désarticulation observées sur les mandibules (récupération de la langue et des joues).

Rachis: les marques de tranchant sur vertèbres indiquent une découpe transversale de la tête au niveau de l'axis ainsi qu'une découpe sagittale du cou opérée par la face ventrale, ce qui implique que l'animal était retourné sur le dos. Le tronçonnement transverse du rachis n'a pas été observé: on remarque seulement une découpe longitudinale de la portion thoracique, effectuée de part et d'autre des épines thoraciques. De manière générale, ces traces irrégulières témoignent d'une découpe peu soignée.

\begin{tabular}{|c|c|c|c|c|c|c|c|}
\hline $\begin{array}{l}\text { Ovicaprinés } \\
\text { Autel }\end{array}$ & $\begin{array}{l}\text { NR (nomb } \\
\text { diaphyse }\end{array}$ & $\begin{array}{l}\text { re de restes) } \\
\text { articulation }\end{array}$ & $\begin{array}{l}\text { NR striés } \\
\text { diaphyse }\end{array}$ & $\begin{array}{l}\text { ou découpés } \\
\text { articulation }\end{array}$ & $\begin{array}{l}\text { \%NR décc } \\
\text { diaphyse }\end{array}$ & $\begin{array}{l}\text { oupe par os } \\
\text { articulation }\end{array}$ & $\begin{array}{l}\text { \%NR striés/ lot } 1 \\
(\mathrm{NR} 0 / \mathrm{C}=189)\end{array}$ \\
\hline Crâne & 27 & & 8 & & 29,6 & & 4,2 \\
\hline Vertèbres & 8 & & 4 & & 50 & & 2,1 \\
\hline Côtes & 49 & 5 & 2 & 0 & 4,1 & 0 & 1,1 \\
\hline Scapula & 3 & 0 & 0 & 0 & 0 & 0 & 0 \\
\hline Humérus & 5 & 2 & 0 & 2 & 0 & 100 & 1,1 \\
\hline Radius & 6 & 4 & 2 & 4 & 33,3 & 100 & 3,2 \\
\hline Ulna & 0 & 0 & 0 & 0 & 0 & 0 & 0 \\
\hline Carpc & 0 & 0 & 0 & 0 & 0 & 0 & 0 \\
\hline Métacarpe & 1 & 3 & 1 & 1 & 100 & 33,3 & 1,1 \\
\hline Coxal & 4 & 5 & 2 & 4 & 50 & 80 & 3,2 \\
\hline Fémur & 0 & 1 & 0 & 0 & 0 & 0 & 0 \\
\hline Tibia & 2 & 2 & 1 & 1 & 50 & 50 & 1,1 \\
\hline Patella & 0 & 0 & 0 & 0 & 0 & 0 & 0 \\
\hline Tarse & 0 & 3 & 0 & 1 & 0 & 33,3 & 0,53 \\
\hline Métatarse & 0 & 3 & 0 & 1 & 0 & 33,3 & 0,53 \\
\hline Phalange & 0 & 2 & 0 & 0 & 0 & 0 & 0 \\
\hline Os longs indét. & 30 & 0 & 7 & 0 & 23,3 & 0 & 3,7 \\
\hline
\end{tabular}

Tableau 2. - Répartition des marques de découpe sur le squelette des moutons (lot 1: autel). 


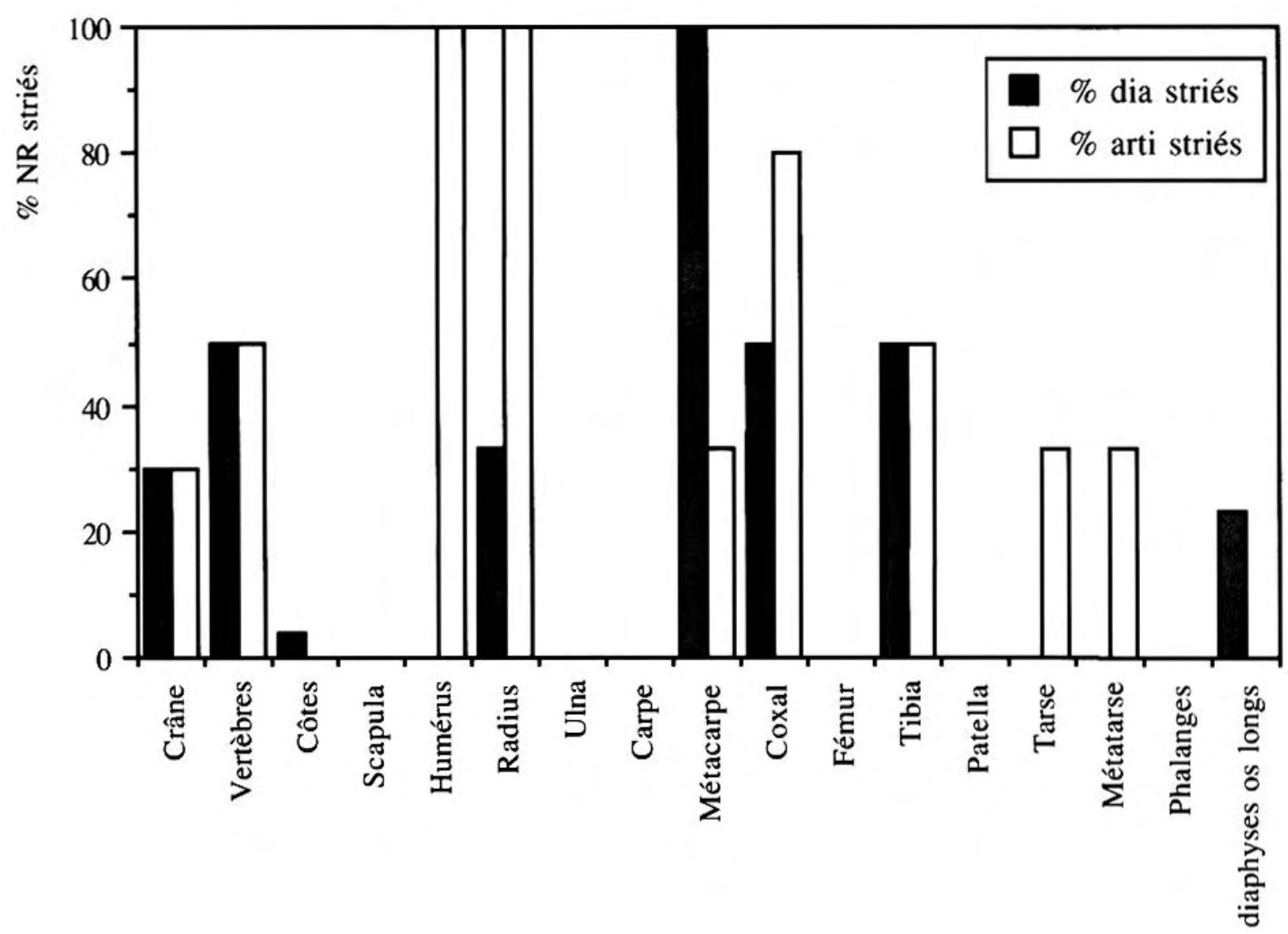

Fig. 4. Nombre de restes osseux porteurs de marques de découpe (moutons).

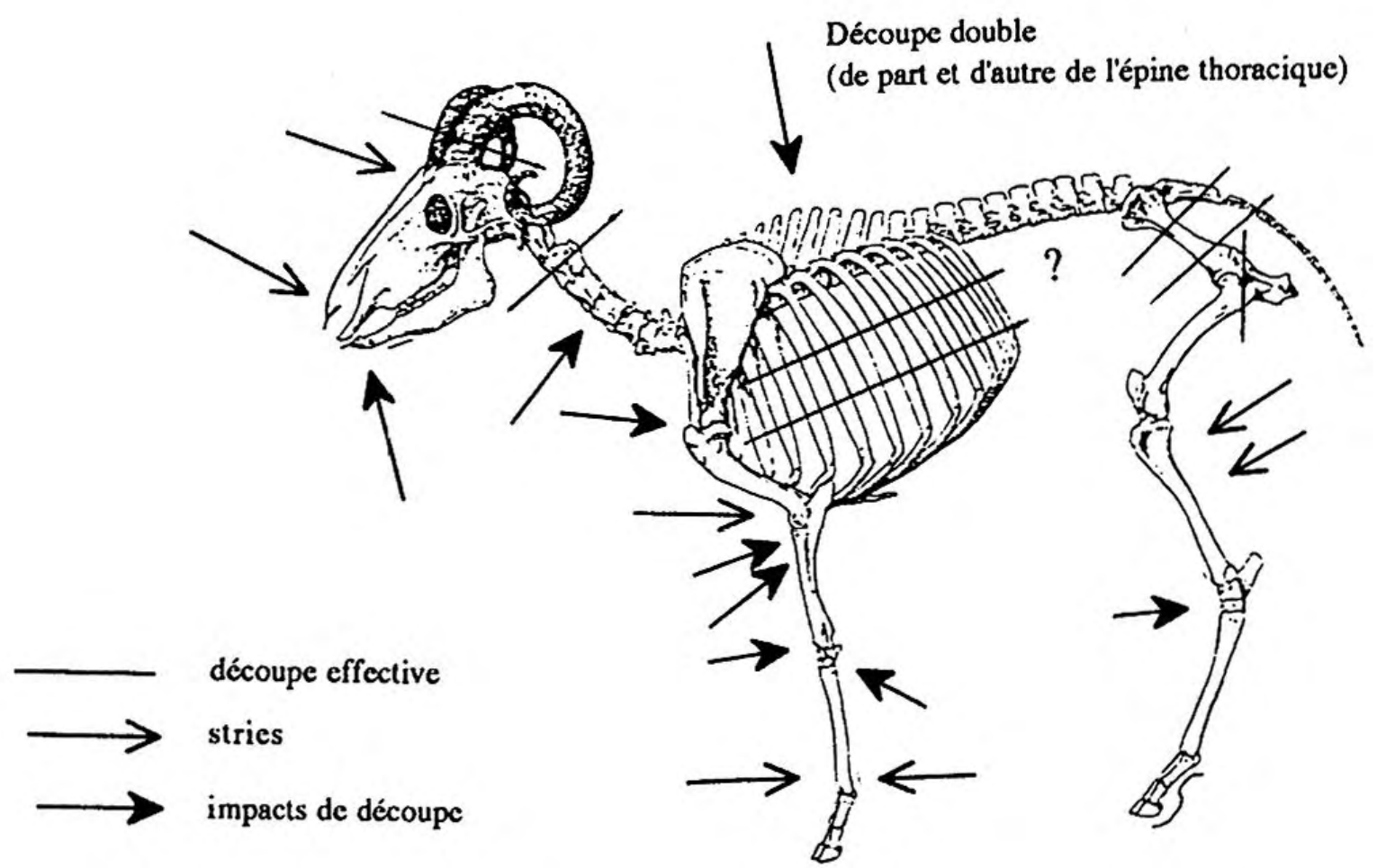

Fig. 5. Localisation et nature des marques de boucherie observées sur les moutons de l'autel. 
En ce qui concerne les côtes, les stries sont très peu nombreuses. Ces ossements ont subi une fragmentation intense qui est sans doute en partie à l'origine de la perte de certains éléments. La rareté des extrémités articulaires peut être mise en relation avec la découpe du rachis thoracique et l'enlèvement des filets, qui auraient entraîné la disparition des connexions entre vertebres et côtes. Cela suppose que ces filets ont dû être consommés et rejetés dans un autre lieu.

Membre antérieur: diverses étapes du débitage des pattes antérieures ont été mises en évidence :

- la découpe et la désarticulation de l'épaule au niveau scapulo-huméral ;

- la désarticulation du coude observée sur la trochlée médiale de l'articulation distale de l'humérus, ainsi que la découpe transverse par la face crâniale du radius sous forme d'impacts de couperet localisés sur la partie proximale de cet os. On remarquera également, sur trois spécimens de radius, des actions de raclage longitudinal de l'os qui se traduisent par des stries et des enlèvements corticaux dans le sens longitudinal et uniquement repérés sur diaphyse. Il est possible que ce type de décarnisation et de nettoyage du radius soit à l'origine du déficit en ulna ;

- la découpe, probablement au couperet, de la base de la patte sous l'articulation du carpe : les impacts transverses sur diaphyse se localisent sur le bord proximal du métacarpe ;

- enfin deux stries résultant d'une découpe circulaire et transversale à la base du métacarpe précisent la technique de dépouillage de l'animal.

Membre postérieur: à l'inverse de la ceinture scapulaire, la ceinture pelvienne est bien représentée par ses os coxaux qui présentent fréquemment des stries à l'intérieur de la cavité acétabulaire, ce qui indique à la fois la découpe et la désarticulation de la patte au niveau du bassin et de la partie proximale du fémur. Cependant, il ne reste que très peu de fémurs pour préciser le sens et les conséquences de cette découpe. Cette remarque va dans le sens d'un traitement particulier des cuisses de moutons (les gigots sont représentés par les fémurs).

La découpe de l'aile iliaque est également observée, qui permet de séparer la " patte " du sacrum, seul point d'ancrage du membre postérieur au squelette.

On notera aussi des petites stries transverses, régulières et fines, sur la face palmaire d'une extrémité proximale de tibia, qui indiquent probablement une action de découpe de portions de viande (découpe en tranches ?). Enfin la découpe de la patte est attestée à la base du tarse par un impact situé sur le bord proximal d'un métatarse.

TABLEAU 2

Bien que les fréquences de restes striés soient relativement faibles, il est possible d'affirmer qu'il y avait dépouillage, puis mise en quartiers de l'animal; le dépeçage est visible au niveau des articulations principales des membres et au niveau du cou. Cette mise en quartiers, apparemment complète, détermine de nombreuses portions de viande mais justifie également la dispersion des restes, puisqu'elle implique un partage de l'animal en vue de sa consommation. Le matériel ostéologique des ovins traduit leur consommation 
partielle, consommation humaine probablement si l'on considère que les viandes sacrifiées au dieu étaient portées sur le bûcher: le taux d'os brûlés n'est en effet que de $2,6 \%$ de l'ensemble du matériel osseux du lot 1 , ce qui est très faible.

Compte tenu du contexte cultuel dont est issu ce matériel, il est possible d'émettre l'hypothèse que les parties manquantes des squelettes ont subi d'autres traitements : éventuelles offrandes au dieu, de type holocaustique, dont il ne reste rien ou seulement de rares os brûlés et conservés de façon anecdotique ou accidentelle (?). Il est alors intéressant de noter que ces portions constituent, selon nos critères actuels, les meilleurs morceaux, à savoir des épaules, gigots, côtes et filets. Nous resterons très prudente, en tout état de cause, face à ces hypothèses qui ne sont que des projections sans fondements ostéologiques, vu que les résidus, même brûlés, de ces offrandes potentielles n'ont pas été retrouvés.

\section{b. Les boeufs du dépôt sacrificiel}

Les restes osseux attribués au bouf constituent approximativement le quart du matériel ostéologique $(23,6 \%$ des restes) et représentent deux individus adultes. Cet ensemble est lui aussi fragmentaire et témoigne d'une dispersion importante des carcasses. Comme dans le cas des moutons, les côtes sont sur-représentées du fait de la fragmentation. La présence des deux individus a été établie sur la base des restes crâniens, au sein desquels on remarquera l'absence totale de dents (d'où le manque de précision dans l'évaluation des âges d'abattage). Le débitage des animaux est attesté par leur taux de restes porteurs de marques, supérieur à celui des moutons : $39,2 \%$ contre $21,7 \%$ chez le mouton. La conservation des portions squelettiques met en évidence la prédominance des restes du FG. 7 rachis, du membre antérieur, du coxal et du métatarse. Ainsi, de même que pour les moutons, certaines parties riches en viande n'apparaissent pas : c'est le cas du membre postérieur et en particulier de la cuisse, qui a manifestement fait l'objet d'un autre traitement.

Enfin, hormis un fragment de métatarse qui témoigne de la découpe de la patte à sa base, aucune extrémité de membre n'a été retrouvée: absence des carpes, métacarpes, tarses et phalanges, ce qui ne peut en aucun cas se justifier par la conservation différentielle ou les techniques de fouille. Leur absence doit donc être considérée comme la preuve d'une exploitation de l'animal en plusieurs étapes, destinations et traitements.

Les profils de représentation anatomique associés à l'analyse des marques de boucherie permettent d'émettre l'hypothèse de deux types de dépôts : des déchets de découpe d'une part (fragments crâniens, coxaux, vertèbres et métatarse) et des déchets de consommation d'autre part (fragments de côtes et du membre antérieur, décharnement des mandibules). Par ailleurs, aucun reste brûlé n'a été répertorié.

Le débitage du bouf s'observe tout d'abord au niveau de l'axis par une découpe transverse qui a séparé la tête du corps, suivie, comme chez le mouton, par une découpe longitudinale du cou également observée sur cette vertèbre. On note ensuite un tronçon- 


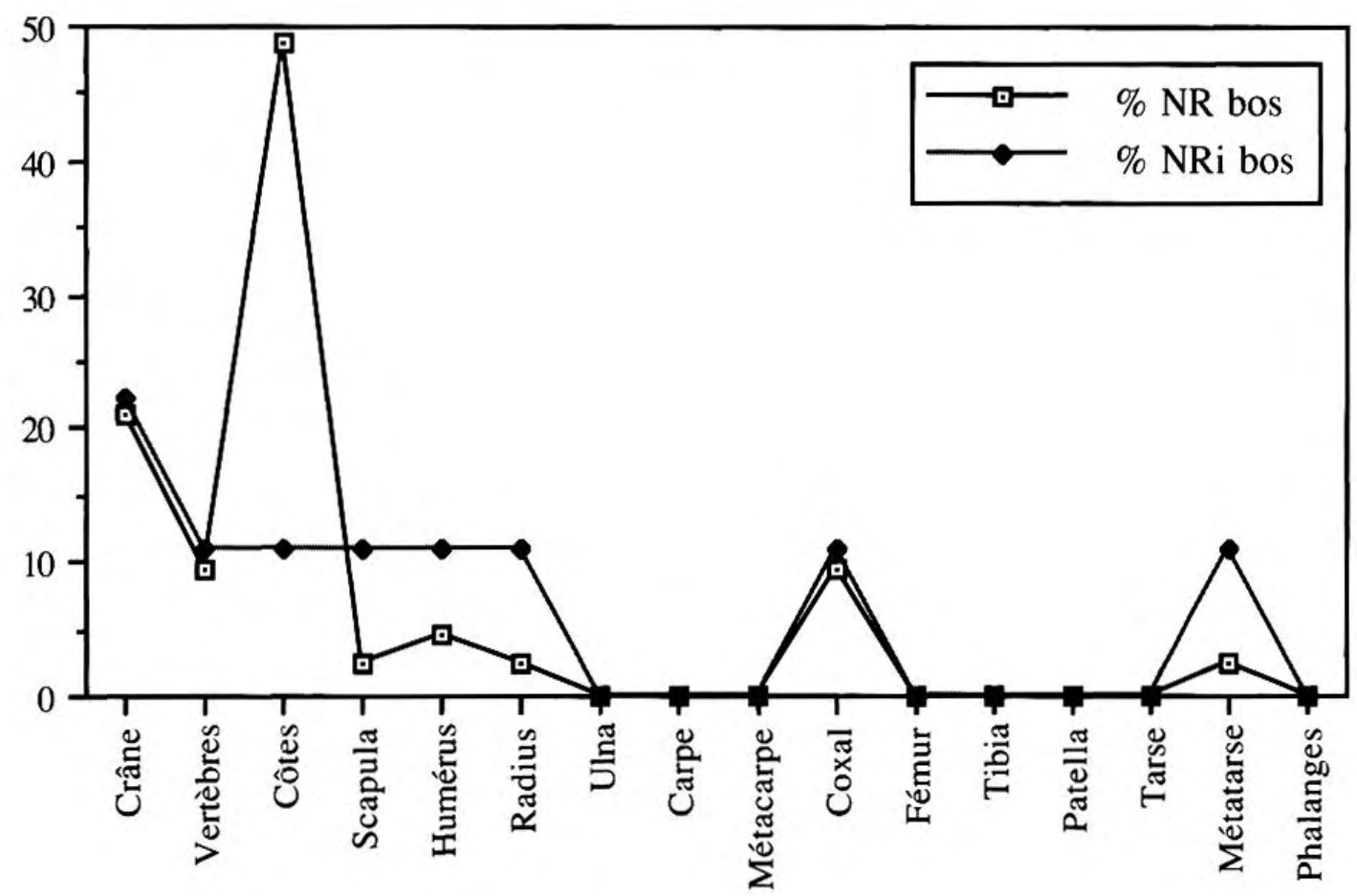

Fig. 6. Représentation anatomique des bcufs de l'autel.

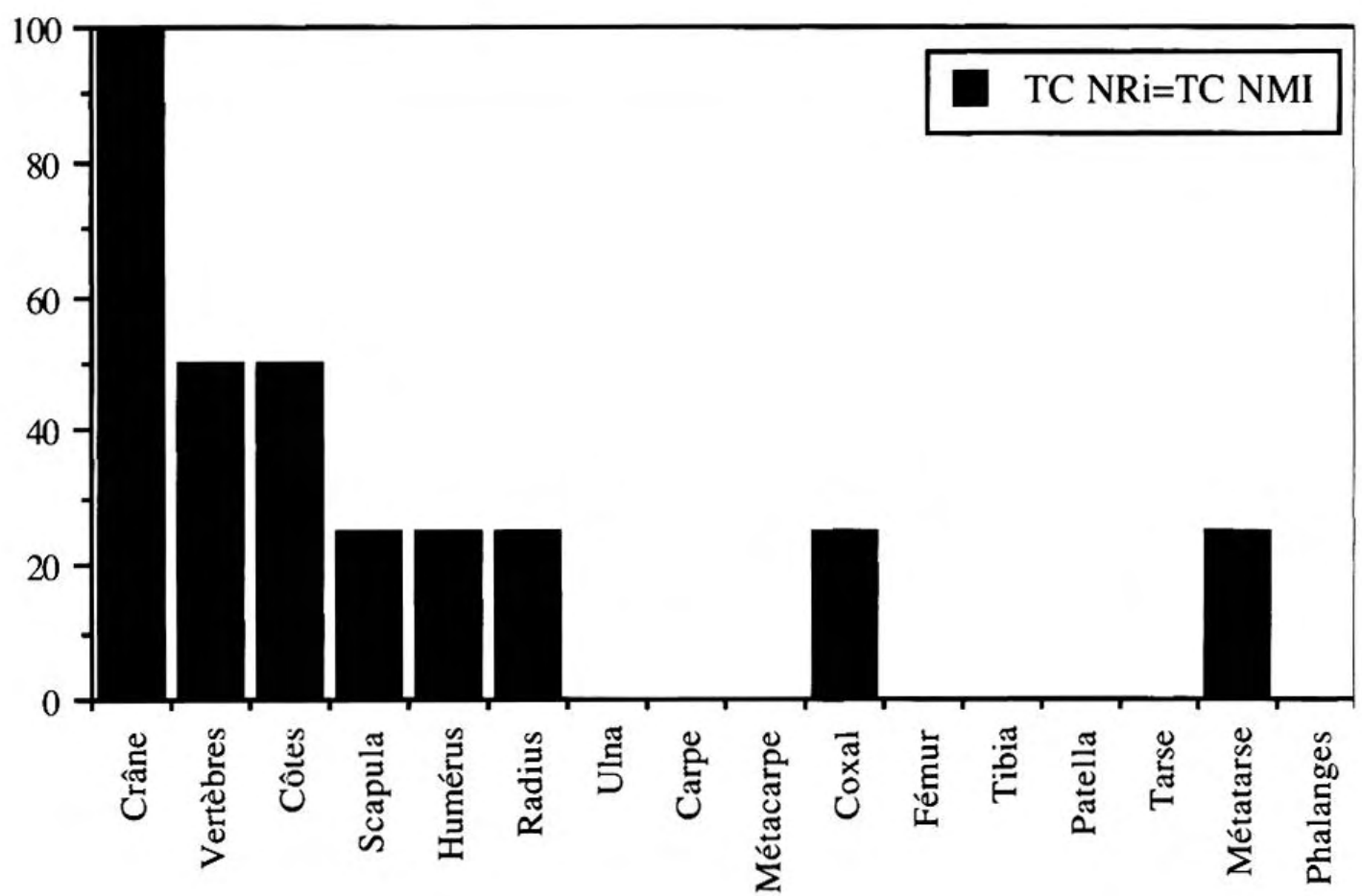

Fg. 7. Taux de conservation des bceufs de l'autel. 
nement du rachis thoracique qui se traduit par des marques de couperet transverses et localisées entre les vertèbres, auxquelles s'associe une double découpe sagittale, de part et d'autre de l'épine thoracique (enlèvement des trains de côtes également observé chez le mouton). Enfin l'élimination ou la découpe de l'extrémité des pattes s'observe à la base du radius ainsi que sur la portion proximale d'un métatarse.

Les témoins plus directs de la consommation effective du bœuf sont les mandibules et les côtes. Les mandibules ont été désarticulées au couperet, entre condyle mandibulaire et os temporal, puis décharnées. Cette dernière opération est visible sur les deux faces de l'os, interne et externe, ce qui suppose l'enlèvement des muscles de la joue ainsi que de la langue.

Les fragments de côtes se caractérisent quant à eux par l'absence d'extrémités articulaires qui ont sans doute été entraînées lors de la double découpe sagittale des vertèbres thoraciques et par la présence de nombreuses portions de corps qui présentent des marques de découpe transverse (mise en quartiers et décharnement des plats de côtes). Ces éléments correspondent plutôt à des restes de repas, déchets de consommation tels qưon les retrouve ordinairement dans les niveaux d'habitat ou dans certains dépotoirs; les fragments de plats de côtes montrent également des stries de décarnisation sur les faces externes et internes, qui peuvent être dûs à la consommation de la viande.

\section{c. Animal sacrifié - Animal consommé}

Dans un premier temps, interpréter le dépôt d'ossements issu de l'espace sacrificiel voué au culte hérakléen implique de s'affranchir du contexte cultuel et de s'en tenir aux observations strictement archéozoologiques.

L'ensemble osseux du dépôt sacrificiel, de par son caractère fragmentaire et son degré de dispersion des squelettes, auxquels s'associe une fréquence d'ossements porteurs de marques de découpe significative dans un contexte taphonomique peu perturbé (peu d'altérations attribuables aux carnivores, au feu ou à des modifications d'origine physico-chimique), évoque des processus de dépôt bien connus, à savoir des déchets de découpe et de consommation. De plus, l'analyse comparative des différents critères taphonomiques relevés au sein des six lots de l'Hérakleion montre que le lot 1 ne s'individualise pas des autres ensembles.

L'analyse fine des caractères extrinsèques et de l'origine anatomique des ossements du dépôt sacrificiel permet alors de faire la différence entre ces deux types de rejet et met en évidence différentes étapes du traitement de l'animal. Ainsi, les opérations de dépouillage (mouton), dépeçage (bœuf et mouton), désarticulation (mouton) et décharnement (bœuf et mouton) ont été observées. Les deux ensembles osseux présentent les mêmes caractéristiques générales de fragmentation, de conservation, de dispersion, mais se distinguent par leurs profils de représentation anatomique qui témoignent du traitement différencié des deux espèces. Selon les cas, certaines parties anatomiques font défaut, voire sont absentes : elles ont été utilisées ou tout au moins rejetées ailleurs. 
La seconde étape de l'interprétation de cet ensemble consiste dans la mise en relation avec les données historiques et épigraphiques. Les travaux les plus récents consacrés à l'Hérakleion de Thasos ${ }^{12}$ confirment la réalité d'un sanctuaire dédié à Héraklès et composé de diverses structures telles que un hestiatorion, un puits, deux temples successifs, une lesché et un autel rupestre en gneiss. Enfin, à l'appui des vestiges architecturaux, la découverte du règlement sacrificicl du Prytanćc permet de préciser une réalité concrète du déroulement du culte, sous forme d'interdits. Le premier de ces interdits exclut du sacrifice les chèvres et les porcs. Il est donc possible de voir une analogie entre ce règlement et le contenu taxonomique du dépôt osseux. Le porc n'est absolument pas présent, mais on le retrouve dans les contextes archaïques des cours 2 , 2bis et 3 , ce qui indique qu'il était consommé à Thasos dans des contextes sans doute domestiques: il y a concordance entre épigraphie et archẻozoologie pour cet animal.

Le cas de la chèvre est un peu plus délicat : en effet, tous les ossements d'ovicaprinés n'ont pas été déterminés spécifiquement et seuls les moutons ont été attestés. Comme dans le cas du porc, on constate que la chèvre est un animal présent dans les niveaux contemporains des cours 2,2 bis et 3 . C'est donc avec une certaine réserve que nous excluons la présence de chèvre dans le dépôt sacrificiel. Il est alors possible d'établir une certaine conformité entre les interdits du rituel et les données archéozoologiques.

Pour ce qui est du traitement des animaux, l'interprétation de déchets alimentaires s'accorde avec les banquets rituels qui accompagnent le culte. Il est également possible de voir, dans la répartition des carcasses, le résultat du partage de l'animal, peut-être entre l'homme et le dieu, mais nos seules données ne permettent pas d'affirmer cette hypothèse dont la confirmation ne pourrait, à notre sens, trouver de réalité que dans la confrontation avec des données textuelles qui nous font malheureusement défaut.

\begin{tabular}{|l|c|c|c|c|c|c|}
\hline & Lot 1 & Lot 2 & Lot 3 & Lot 4 & Lot 5 & Lot 6 \\
\hline DÉcouPE & & & & & & \\
Ovicaprinés & 21,7 & 17,4 & 27,3 & 23,1 & 4,9 & 10,9 \\
Bœuf & 39,2 & 40 & 14,3 & 41,4 & 22,2 & 47,7 \\
Porc & 0 & 0 & 0 & 50 & 50 & 20 \\
\hline NR Brûlés & 2,6 & 13,3 & 2,3 & 0 & 34,7 & 27,2 \\
\hline NR Carnivores & 0,9 & 0 & 4,6 & 0 & 1,8 & 2,4 \\
\hline NR Concrétions & 6,4 & 0 & 0 & 0 & 0 & 0 \\
\hline Total NR lot & 235 & 30 & 43 & 116 & 167 & 254 \\
\hline
\end{tabular}

Tableau 3. - Pourcentage des restes osseux porteurs de marques d'origine humaine, animale ou physico-chimique. N.B. : tous les restes concrétionnés sont issus de la couche 85.507 . 


\section{Ossements indirectement associés au dépôt de l'autel (lot 2)}

Ce petit ensemble faunique, malgré un nombre de restes restreint, présente des similitudes avec le précédent : seuls sont représentés le bœuf et le mouton. Il s'agit d'un bœuf adulte et de deux ovins : le premier est estimé à environ 1 an et le second entre 4 et 5 taBleau 1 ans. Les ossements témoignent d'une activité de découpe et de consommation qui s'accorde avec les données observées dans le lot 1 : deux ustensiles ont été reconnus, à savoir le couperet, qui provoque une découpe franche et nette de l'os et le couteau, utilisé principalement lors de la désarticulation, du décharnement et de la découpe de portion de viande, qui ne laisse que des stries fines et peu profondes. Bien que l'association archéologique entre ce petit lot et celui de l'autel soit douteuse chronologiquement ou archéologiquement, force est de constater que leur nature archéozoologique les rapproche.

\section{Remblais du temple B (lot 3)}

Le matériel faunique issu des remblais du second temple ou temple B est contemporain de la construction de ce dernier, dans la $2^{\mathrm{e}}$ moitié du $\mathrm{V}^{\mathrm{e}}$ siècle av. J.-C. Une nouvelle fois, la même remarque s'impose concernant ces assemblages " archaïques " associés aux temples de l'Hérakleion : seuls les ovicaprinés, toujours dominants, et les bœufs sont présents. Cependant, à la différence des deux lots précédents, l'état de fragmentation des

tABleau 1 restes n'a pas permis de diagnose différencielle entre Ovis et Capra.

Statistiquement, cette série est relativement faible et donc délicate à interpréter. La fréquence des marques de découpe indique un traitement intensif des animaux alors que les autres agents susceptibles de modifier la nature de l'assemblage restent discrets: feu, TABLEAU 3 carnivores, altérations physico-chimiques. Les rejets osseux correspondent ici à des parties peu riches en viande (si on exclut les restes d'un cou de bœuf découpé), avec une dominance des fragments crâniens et particulièrement des mandibules (les têtes sont séparées du corps au niveau cervical) sur les restes du squelette appendiculaire. On constate néanmoins une bonne représentation des ceintures pelviennes : les coxaux sont des os qui résistent bien aux altérations post-dépositionnelles. Les extrémités des membres sont absentes à partir des articulations carpiennes et tarsiennes. Il peut alors s'agir de déchets de débitage mêlés, avec des reliefs de repas, au sédiment employé dans les remblais du temple.

\section{Restes fauniques des sondages cour 2, 2bis et 3 (lot 4)}

Les fouilles des cours 2 , 2bis et 3, attenantes au complexe du sanctuaire d'Héraklès ont livré un matériel faunique contemporain de la période archaïque. Les niveaux supérieurs, remaniés et plus récents, ont été éliminés de l'analyse. 
Ce lot a fourni un matériel osseux et dentaire qui confirme, hors contexte directement associé au culte, la dominance du cheptel ovin et caprin puis du cheptel bovin dans l'économie pastorale thasienne. On constate cependant que le spectre faunique s'élargit à deux nouvelles espèces: le porc et un oiseau de type galliforme, la présence de la tortue n'étant pas directement associée à l'assemblage d'origine anthropique, même si sa consommation n'est pas exclue.

La population ovine-caprine est constituée d'un très jeune individu de moins de six mois, d'un agneau (ou chevreau) de 6 à 12 mois, d'un jeune adulte de 18 à 24 mois et de deux individus de 24 à 36 mois. Malgré la faiblesse de l'échantillon, il semble ne pas y avoir choix d'un âge d'abattage précis, mais prélèvement hétéroclite qui témoigne soit d'un goût diversifié soit de différentes saisons d'abattage.

Parmi les bœufs, on note la présence d'un individu juvénile (entre 6 et 18 mois) et d'un adulte (entre 4 et 8 ans). Ces dernières classes d'âge ont été établies à partir des stades d'usure dentaire. Enfin, deux fragments de tibia issus des deux couches $\mathrm{N}^{\circ} 628$ et $\mathrm{N}^{\circ} 636$ appartiennent au même os dont l'état d'épiphysation de l'extrémité distale indique une fourchette de 24 à 30 mois.

Dans l'ensemble, les ossements porteurs de marques de découpe sont peu nombreux et la fréquence des os brûlés est la plus importante des niveaux archaïques de l'Hérakleion. Pourrait-il s'agir de vidanges de foyers?

Ce lot très hétérogène et témoin d'une dispersion importante des restes présente toutefois l'avantage de mettre en évidence l'existence sur le site du porc, malgré une fréquence très réduite de ses restes $(1,8 \%$ du NR) et d'un oiseau assimilable à une volaille domestique (cf. Gallus sp.), cette détermination restant sous réserve.

\section{Restes fauniques issus des cours 4 et 4 bis (lot 5 )}

Cet assemblage est assimilable à celui des cours 2,2 bis et 3, mais a été traité isolément du fait de sa localisation topographique. Le spectre faunique est sensiblement le même que dans les autres cours, du fait de la dominance des ovins-caprins sur le bœuf, le porc puis la volaille.

Les marques de boucherie, en particulier sur les ovicaprinés, évoquent un débitage qui a entraîné l'élimination des ossements appartenant à l'extrémité des membres. De même, les os longs du membre antérieur, humérus et radius, montrent la désarticulation et la découpe de la patte en portions probablement prédéterminées. 


\section{Restes fauniques issus de la "favissa " (lot 6)}

L'assemblage osseux exhumé lors de la fouille de la favissa était associé à de la céramique hellénistique qui tient lieu de marqueur chronologique. Ce lot s'individualise des autres ensembles par son contexte archéologique, sa chronologie, et surtout un spectre faunique qui fait apparaître une population de porcs dépassant celle des bovins et accompagnée d'un équidé (âne ou cheval de petite taille).

Les restes osseux sont bien conservés et ont été relativement épargnés par la frag-

TABLEAU 1

TABLEAU 3

TABLEAU 1 mentation, ce qui a permis d'atteindre un taux de détermination supérieur à $90 \%$. De plus, ce matériel ne présente aucune trace d'altération telle que l'action du feu ou des carnivores.

\section{a. Les ovicaprinés}

La population d'ovicaprinés est estimée à neuf individus qui se partagent entre 5 moutons et 4 chèvres, déterminés selon les critères classiques de différenciation morphologique $^{13}$, à partir d'une série de seize métapodes (métacarpes et métatarses) entiers. Néanmoins, on notera que l'analyse biométrique n'a pas permis d'isoler aussi nettement les populations. Ces ossements sont également à l'origine de la détermination des âges d'abattage qui ont mis en évidence la présence de deux individus de moins de 18 mois (un mouton et une chèvre), d'un mouton de 18 à 24 mois et de six individus de plus de 24 mois (trois moutons et trois chèvres) : la majorité des bêtes est donc abattue à l'âge adulte.

Les restes présentent des traces de découpe bouchère fréquentes, qui permettent d'établir un schéma d'exploitation des carcasses : la tête, le rachis et les ceintures, en particulier pelvienne, ont fait l'objet d'un débitage pratiqué au couperet. Les os longs présentent des stries de raclage ou de découpe/désarticulation effectuées au couteau. La particularité de cet assemblage est d'être essentiellement constitué de métapodes entiers (NR = 16/62 restes d'ovicaprinés), alors que les autres ossements sont tous fragmentés: ils témoignent donc soit d'un dépôt spécifique, soit d'une technique de découpe particulière. Dans les deux cas, la nature même de cet os qui ne porte que très peu de chair consommable et dont ses caractéristiques morphologiques font un élément souvent utilisé pour l'artisanat oriente vers un dépôt de type "déchets de découpe ". A l'inverse, les autres éléments du squelette ont probablement été consommés comme l'indiquent les marques de découpe : la tête est découpée au niveau de l'axis, les chevilles sont éliminées et les mandibules tranchées à la symphyse lorsque que la tête est retournée. Le cou est débité transversalement et sagittalement; les vertèbres thoraciques sont découpées longitudinalement de part et d'autre de l'épine, ce qui correspond à l'enlèvement des côtes; celles-ci ne présentent pas de traces et la découpe du rachis thoracique devait entraîner leurs extrémités articulaires avec les filets. 
L'assemblage met en évidence une population qui témoigne d'un dimorphisme spécifique et sexuel marqué ; malheureusement les seules séries d'os mesurables se limitent aux métapodes qui ne sont pas assez nombreux pour atteindre un degré de validité statisTABLEAU 4 tique satisfaisant.

\section{HUMÉRUS}

\begin{tabular}{|l|c|c|c|c|}
\hline Lot & US / FAIT & DT prox & DT dist & DAP dist \\
\hline 1 & autel & & & 48,6 \\
1 & autel & 30,6 & 28,2 & \\
6 & 84.2057 & 30,1 & 25 & \\
4 & $85.602 .1 \mathrm{a}$ & 31,4 & 27,1 & \\
4 & $85.602 .1 \mathrm{a}$ & 29,5 & 26,6 & \\
\hline
\end{tabular}

\section{RADIUS}

\begin{tabular}{|l|c|c|c|c|c|}
\hline Lot & US / FAIT & DT prox & DAP prox & DT dist & DAP dist \\
\hline 1 & autel & 33,3 & 16,3 & & \\
1 & autel & 31,1 & 15,8 & & \\
1 & autel & 30,8 & 15,6 & & \\
1 & autel & & & 30,2 & 20,1 \\
6 & 84.2057 & 34,2 & 18,4 & & \\
6 & 84.2057 & & & 32,3 & 20,5 \\
5 & 85.728 & 34,2 & 16,3 & & \\
\hline
\end{tabular}

\section{MÉTACARPE}

\begin{tabular}{|c|c|c|c|c|c|c|c|c|c|}
\hline Lot & US / FAIT & $L \max$ & DT prox & DAP prox & DT dia & DAP dia & DT dist & DAP dist & $N^{\circ}$ de l'os \\
\hline 1 & autel & 123,2 & 24 & 17 & 9,1 & & 26,4 & 16,5 & \\
\hline 1 & autel & & 24,4 & 17,5 & & & & & \\
\hline 1 & autel & & 25,5 & 17,7 & & & & & \\
\hline 6 & 84.2057 & 127,8 & 24,7 & 17,7 & 15,1 & 11,3 & 27,5 & 16,8 & 7 \\
\hline 6 & 84.2057 & 144,5 & 27,9 & 20,1 & 17,2 & 11,9 & 30,8 & 18,5 & 8 \\
\hline 6 & 84.2057 & & 28,6 & 22 & 16 & 12,5 & & & 9 \\
\hline 6 & 84.2057 & & 27 & 18,6 & 15,7 & 11,9 & & & 10 \\
\hline 6 & 84.2057 & 146,2 & 25,3 & 17,7 & 16,7 & 12,3 & 29,4 & 19,2 & 11 \\
\hline 6 & 84.2057 & & 25,8 & 18,8 & & & & & 12 \\
\hline 6 & 84.2057 & 123,3 & 27,1 & 18,5 & 16,2 & 12 & 28,4 & 18,2 & 13 \\
\hline 6 & 84.2057 & 114 & 24,7 & 17,6 & 14,6 & 10,9 & 25,9 & 15,3 & 14 \\
\hline 6 & 84.2057 & & 25,1 & 17,9 & 17,4 & 11,8 & & & 15 \\
\hline 6 & 84.2057 & 101,7 & 21,8 & 15,8 & 13,9 & 9,9 & 23,4 & 13,8 & 16 \\
\hline 4 & 85.601 .13 & & 26,4 & 18,6 & & & & & \\
\hline 5 & 85.731 & & 24,9 & 18,2 & & & & & \\
\hline
\end{tabular}

\section{MÉTATARSE}

\begin{tabular}{|l|c|c|c|c|c|c|c|c|c|}
\hline Lot & US / FAIT & L max & DT prox & DAP prox & DT dia & DAP dia & DT dist & DAP dist & $N^{\circ}$ de I'os \\
\hline 6 & 84.2057 & 148,2 & 21,1 & 21,2 & 12,1 & 11,8 & 24,1 & 16,6 & 1 \\
6 & 84.2057 & 127,5 & 19 & 18,9 & 11,5 & 10,6 & & & 2 \\
6 & 84.2057 & 163 & 23,1 & 23,3 & 14,5 & 13,1 & & 16,4 & 4 \\
6 & 84.2057 & 139,8 & 22 & 21,5 & 14,4 & 12,1 & 25,9 & 16,4 & 5 \\
6 & 84.2057 & 130,6 & 22,6 & 22,3 & 13,9 & 14 & 26,1 & 17 & 5 \\
\hline
\end{tabular}

\section{TIBIA}

\begin{tabular}{|l|c|c|c|}
\hline Lot & US / FAIT & DT dist & DAP dist \\
\hline 1 & autel & 26,5 & 21,7 \\
6 & 84.2057 & 26,8 & 20,9 \\
4 & $85.602 .1 \mathrm{a}$ & 25,4 & 20,2 \\
4 & $85.602 .1 \mathrm{a}$ & 28,1 & 20,4 \\
\hline
\end{tabular}

\section{CALCANÉUM}

\begin{tabular}{|l|cc|c|}
\hline Lot & US / FAIT & L max & DT max \\
\hline 5 & 85.730 & 56,8 & 20,8 \\
\hline
\end{tabular}




\section{b. Les porcs}

Quatre porcins ont été dénombrés à partir des restes d'hémi-mandibules qui constituent l'essentiel de cet assemblage. Le squelette post-céphalique est en effet très peu représenté et ces éléments épars appartiennent surtout à des individus juvéniles. La conservation privilégiée de ces restes crâniens correspond à un stade précis de la préparation des porcs, qui consiste en la récupération et l'exploitation de leurs têtes. Aucun critère de choix n'est déterminable sur une si petite population : le premier individu a entre 6 et 8 mois, le second de 14 à 17 mois, le troisième entre 7 et 10 ans, et enfin le quatrième est une truie de plus de dix ans, probablement laissée en vie jusquà cet âge en raison de ses qualités reproductrices. Les têtes ont été découpées longitudinalement, puis les maxillaires décharnés et enfin les mandibules désarticulées. Quelques traces observées sur le post-céphalique indiquent que le reste du squelette n'était pas négligé mais plutôt distribué ailleurs.

\section{c. Grands herbivores}

Les grands herbivores tels que équidés et bovidés sont représentés par quelques restes osseux ou dentaires qui témoignent d'opérations de débitage relatives à leur consommation, sans toutefois que celle-ci semble aussi systématique que dans le cas des petits herbivores. On notera tout particulièrement les restes de plats de côtes qui indiquent que ces portions étaient probablement appréciées.

L'assemblage faunique de la " favissa " permet d'obtenir un aperçu des modes et des choix alimentaires thasiens à lépoque hellénistique. Elle s'oppose donc par sa chronologie et son spectre faunique aux lots précédents issus de contextes archaïques associés au sanctuaire hérakléen. 
* Abréviations bibliographiques: BARONE (1986) = R. BARONE, Anatomie comparée des animaux domestiques. I. Ostéologie (1986)

BOESSNECK, MÜLLER, TEICHERT (1964) = J. BOESSNECK, H.H. MÜLLER, M. TEICHERT, - Osteologische Unterscheidungsmerkmale zwishen Schaf (Ovis aries Linné) und Ziege (Capra hircus Linné) ", Kühn-Archiv 78 (1964), p. 1-129

DES COURTILS $(1993)=J$. DES COURTLLS, Architectures thasiennes: recherches sur l'architecture monumentale de Thasos et de l'Egée du Nord à l'époque archaique et classique, Thèse de Doctorat d'Etat, Université de Paris X - Nanterre (1993) DES COURTILS, PARIENTE (1991) $=J$. DES COURTILS, A. PARIENTE, "Problèmes topographiques et religieux à l'Hérakleion de Thasos *, in L'espace sacrificiel dans les civilisations méditerranéennes de I'Antiquité, Publications de la Bibliothèque Salomon-Reinach, Universite Lyon 2, V (1991), 0. 67.73

LAUNEY (1951) = M. LAUNEY, Le sanctuaire et le culte d'Heraklès à Thasos, Etthas I (1951)

PAYNE (1973) = S. PAYNE, "Kill-off patterns in sheep and goats : the mandibles from Asvan Kale ", Anatolian Studies 23 (1973), p. 281303

PICARD (1923) $=\mathrm{Ch}$. PICARD, * Un rituel archaïque d'Héraklès thasien trouvé à Thasos *, BCH 47 (1923), p. 241-274. SEYRIG (1927) $=H$. SEYRIG, BCH 51 (1927), p. $193-198$

VERBANCK-PIERARD $(1989)=$ A. VERBANCK. PIERARD, "Le double culte d'Héraklès : légende ou réalité ", Lire les polythéismes II (1989), p. 3865.

Abréviations :

NR: nombre de restes (indifféremment dents ou ossements).

NRi : nombre de restes initial : correspond a une estimation, au même titre que le NMI, du nombre d'éléments (ou os) entiers originellement présents dans l'assemblage fauniquc, cquivalent du NME anglo-saxon. NMI : nombre minimum d'individus (ici NMI de combinaison).

TC : taux de conservation.

O/C : ovin/caprin.
1 Picaro (1923); SeYrig (1927).

2 Fouilles de 1932 à 1934, publication : LAUNEY (1951).

3 Fouilles effectuées par J. des Courtils et

A. Pariente en 1984 et 1985 , voir les rapports provisoires $B C H 109$ (1985), p. $881-884 ; 110$ (1986), p. 802-809. Lors des fouilles de $M$. Launey, des ossements analogues ont dû être découverts (mais n'ont pas été conservés) : aussi bien avons. nous retrouvé en 1984 et 1985 des tessons provenant de vases dont Launey avait découvert d'autres fragments. 4 DES COURTILS, PARIENTE (1991).

5 On en trouve également dans le lot 4, mais ce dernier est peutêtre formé de remblais antérieurs à l'installation du sanctuaire, comme l'indiqueraient des tessons de vases non tournés datant peutêtre de la phase précoloniale.

$6 \mathrm{~J}$. PoullLoux, Recherches sur l'histoire et les cultes de Thasos I, EtThas III (1954), p. 77 ; J. BOUSQUET, BCH 83 (1959), p. 398. Dans notre étude de 1991, nous avions admis la possibilité d'héberger les deux cultes dans l'Hérakleion, nous fondant sur I'attestation à Thasos de fêtes en I'honneur des héros et comportant des banquets : or l'existence de banquets est bien attestée à l'Hérakleion, comme on l'a vu. Cependant, les banquets dont on y a trouvé les restes ne respectent pas les interdits du texte rituel * du prytanée * : il faut donc bien qu'il ait existé un autre lieu de culte où ce rituel fût observé.

7 Les affinités orientales du dieu en seraient alors la meilleure explication, par opposition à l'Héraklès grec qui, lui, bien qu'héroïque par nature, aurait finalement reçu le même traitement que les autres dieux. L'attitude de VERBANCK.PIERARD (1989) est beaucoup plus tranchée et exclut totalement la possibilité d'un culte héroïque.

8 DES COURTLLS (1993).

9 PICARD (1923).

10 PAYNE (1973).

11 BARONE (1986).

12 DES COURTILS, PARIENTE (1991); DES COURTILS (1993).

13 BOESSNECK, MŨLLER, TEICHERT (1964). 